\title{
Cutaneous vasculitis
} preceding the onset of anti-GBM disease (Goodpasture syndrome)

\section{Vascularite cutanée comme première manifestation des anticorps anti-MBG (syndrome de Goodpasture)}

Anti-glomerular basement membrane disease (anti-GBM-disease, formerly Goodpasture's disease) is an organ-specific immune complex small vessel vasculitis affecting glomerular and/or pulmonary capillaries with GBM deposition of anti-GBM autoantibodies $[1,2]$. Symptoms include arthralgias, fever, rapidly severe glomerulonephritis and/or alveolitis with possibly lung hemorrhage $[2,3]$. The main differential diagnosis includes mainly ANCA associated vasculitis. We report here the unusual case of a woman who presented cutaneous leucocytoclastic vasculitis 4 months prior to anti-GBM disease. To our knowledge, anti-GBM-disease has not been associated with cutaneous small vessel vasculitis.

\section{Observation}

In September 2017, a 63-year-old woman was referred by a private dermatologist for petechial purpura of the lower limbs and joint pain on the ankles. Symptoms had started 3 weeks before referral. Her past medical history included palmo-plantar pustulosis in the 1980-1990s, psoriatic arthritis, and post-surgical hypothyroidism. Her more recent history was notable for right kidney hydronephrosis due to a stenosis of pyelo-ureteral junction treated by pyeloplastia in the end of 2016 with improvement of the kidney function and right urolithiasis managed by extracorporeal shock wave lithotripsy in March 2017. A skin biopsy performed prior to referral on a petechia showed features consistent with small vessel leucocytoclastic vasculitis. No direct immunofluorescence (DIF) was performed. The patient had already received a two-week course of oral corticosteroids (CS, prednisolone $40 \mathrm{mg} /$ day 1 week and $20 \mathrm{mg} /$ day 1 week). She presented mainly post-inflammatory pigmentation of the lower limbs and discrete petechial purpura. She did not have any other cutaneous or mucosal lesions favoring vasculitis (necrosis, nodules, livedo, ulcer...). Physical examination was mainly normal. She did not had any respiratory symptoms, nor edema of the lower limbs especially. She reported moderate pain on the ankles without arthritis and a recent bilateral nose bleeding. Arthralgia was considered to be associated with vasculitis. She had no sign of active psoriatic arthritis, psoriasis or pustulosis. In October, an ENT specialist failed to find any lesion for nose bleeding. Nasal mucosal vasculitis was ruled out. As purpura subsided, DIF was not performed in absence of fresh lesion and oral CS were not initiated again. She was asked to rest and use compressive stockings. Most laboratory findings (serum protein electrophoresis, antinuclear antibodies, ANCA, rheumatoid factor, cryoglobulin, complement component C3 and C4, HIV, hepatitis $B$ and ( serology) were negative or within normal ranges. Only anti-streptolysin $(1040 \mathrm{lU} / \mathrm{mL})$ were elevated. Renal function was normal (creatinine $55 \mathrm{mmol} / \mathrm{L}$, glomerular filtration rate [GFR] $96 \mathrm{~mL} / \mathrm{min} / \mathrm{m}^{2}$ ) but the patient disclosed proteinuria $(1.78 \mathrm{gr} / 24 \mathrm{~h})$ associated with microscopic hematuria and leucocyturia. There were not previous values to compare with. Nephrologist and urologist were consulted but no further explorations were performed because of hydronephrosis and recent history of urolithiasis. In January 2018, the patient developed an acute fever, dry cough associated with oliguria and several renal failure (serum creatinine $820 \mathrm{mmol} / \mathrm{L}$; urea $31 \mathrm{mmol} / \mathrm{L}$; GFR $4 \mathrm{~mL} / \mathrm{min} / \mathrm{m}^{2}$ ). Indirect IF test showed anti-GBM circulating antibodies and anti-GBM antibodies against the NC1 domain of the alpha 3 chain of type IV collagen detected by immunoassay were highly positive $>680 \mathrm{U} / \mathrm{mL}$. ANCA were controlled and both anti-MPO and anti-PR3 antibodies were negative. No kidney biopsy was performed. The patient was managed by high-dose CS, cyclophosphamide, plasmapheresis and dialysis.

\section{Discussion}

We report here the unusual case of a woman who presented a cutaneous leucocytoclastic vasculitis 4 months prior to the onset of anti-GBM disease. The patient presented also urinalysis anomalies that could have indicated the onset of anti-GBM disease. Past urinary history of the patient and lack of renal dysfunction prevented to explore further the patient at the time. We failed to find in the literature any case of anti-GBM disease associated with skin vasculitis. Reviews do not mention cutaneous vasculitis as a manifestation of the disease $[2,3]$. The main differential diagnosis would have been another smallvessel vasculitis such as ANCA-associated vasculitis or lupus. However, the patient did not have any circulating antibodies. Interestingly, the possible role of lithotripsy as an environmental factor for anti-GBM disease has been reported in anecdotal cases [4]. Here lithotripsy was performed 10 prior to onset of the 
disease. We cannot naturally rule out a coincidental association of two unrelated small vessel vasculitis. Palmo-plantar pustulosis and psoriatic arthritis are usually not associated with skin vasculitis and both conditions had been in remission for years. The patient displayed high levels of anti-streptolysin so that she may have had a transient post-streptococcal vasculitis. Lastly, cutaneous vasculitis associated with the specific past renal history of the patients would have acted as potential triggering factors through microscopic insult in the lung or kidney capillaries leading to anti-GBM disease.

Because of the severity of anti-GBM-disease, we thought it was opportune to report this case. Beside, it acts as a reminder that urinalysis anomalies in patients with cutaneous small vessel vasculitis should be explored or followed thoroughly. Preexisting urologic or renal conditions should not mislead to neglect a possible renal vasculitis.

Funding sources: none.

Disclosure of interest: the author declares that he has no competing interest.

\section{References}

[1] Jennette JC, Falk RJ, Bacon PA, Basu N, Cid MC, Ferrario F, et al. 2012 revised International Chapel Hill Consensus Conference Nomenclature of Vasculitides. Arthritis Rheum 2013;65:1-11.

[2] Hellmark T, Segelmark M. Diagnosis and classification of Goodpasture's disease (anti-GBM). J Autoimmun 2014;48-49:108-12.

[3] Greco A, Rizzo MI, De Virgilio A, Gallo A, Fusconi M, Pagliuca G, et al. Goodpasture's syndrome: a clinical update. Autoimmun Rev 2015;14:24653.

[4] Xenocostas A, Jothy S, Collins B, Loertscher R, Levy M. Anti-glomerular basement membrane glomerulonephritis after extracorporeal shock wave lithotripsy. Am J Kidney Dis 1999;33:128-32.

Nicolas Kluger

University of Helsinki, Helsinki University Central Hospital, Departments of dermatology, allergology and venereology, Meilahdentie 2, P0 Box 160, 00029 Helsinki, Finland

nicolas.kluger@hus.fi

Received 20 May 2018 Accepted 7 November 2018 Available online:

https://doi.org/10.1016/j.lpm.2018.11.001

(c) 2018 Elsevier Masson SAS. All rights reserved. 